\title{
LAMARCKISME ET SOLIDARITÉ ${ }^{1}$
}

\author{
Antonello La Vergata
}

Istituto e Museo di Storia della Scienza di Firenze

En présentant l'ouvrage de Robert Tocquet L'entraide dans le monde des animaux et des plantes (1953), Louis Marin, de l'Institut écrivait que "le thème et la façon de le développer [étaient] dans la pleine tradition de notre Pays", car le livre contenait "des conceptions philosophiques conformes à notre génie national". "Si la Science n'a pas de patrie", continuait-il en répétant une opinion célèbre de Pasteur, "les savants en ont une".

"Avec des méthodes de découverte universelles, avec un langage de plus en plus semblable pour les exposer, les savants (les vrais beaucoup plus que les médiocres) sont marqués parle terroir. Dans cette immense question de l'évolution de la vie et de la formation des espèces, les grands naturalistes ont été guidés par les tendances de leur peuples; en tout cas,sur le sujet spécial de ce livre - le rôle de l'entraide et de la lutte pour l'existence- ils se classent manifestement suivant leur origine nationale.

En faveur de la prédominance de l'entraide au cours du développement des êtres vivants et, particulièrement, des animaux, les Français ont précédé les autres et, dès l'organisation des études méthodiques au XVIIIe siècle - de Rousseau, Daubenton, d'Orbigny, jusqu'à Espinasse [sic], Lanessan, Blanchard, Fabre, Maeterlinck, Delamain - ont développé des doctrines de fraternité. A leur encontre, les maîtres anglais en la matière, avec Hobbes, Darwin, Spencer, Wallace, Huxley ont personnifié et propagé la thèse la plus cruelle de la lutte pour la vie. Les Allemands les ont suivis pour la plupart; mais, selon l'usage, quelquesuns ont prôné, au contraire, avec lyrisme, le rôle de l'amour dans la Création. Les Russe avaient d'abord adoptés les doctrines anglo-allemandes; mais leur nature tolstoïsante les ramena bientôt à nos côtés ${ }^{2}$."

Marin était d'accord avec l'auteur de l'ouvrage qu'il présentait:

«...Dans l'organisation et l'essor de la Vie, les ressources de l'entraide, conformément aux doctrines françaises et contrairement à ce qu'ont clamé les évolutionnaires anglais à propos de la Lutte pour la Vie et de la Loi de la Jungle qui en découle, ont joué un rôle infiniment plus important et, surtout, plus fécond que la force: même au sein des sociétés anima-

1 Texte d'une communication lue au colloque pour le bicentenaire du Muséum National d'Histoire Naturelle (Paris, juin, 1993), à paraitre dans les Actes du colloque.

2 Marin, L. (1953), Introduction à Tocquet, 1953, pp. V-VI. 


\begin{abstract}
les, les régimes de liberté ont été des ferments supérieurs à ceux de la brutalité. Le maître des écrivais - et le plus populaire - a bien rendu la pensée du bon sens français:

Il se faut entraider: c'est la loi de Nature.»3${ }^{3}$
\end{abstract}

Nous n'avons pas l'intention de nous livrer ici à une enquête d'ethnopsychologie de la biologie. Nous nous bornerons à souligner le rapport étroit existant entre néolamarckisme et éloge de l'entraide en France.

On sait que les néolamarckiens se refusaient d'attribuer à la sélection naturelle le rôle principal danss l'évolution. Il est peut-être moins connu qu'ils avaient une conception différente de la lutte pour la vie par rapport à celle de Darwin. Dans leur interprétation la lutte était un stimulant pour le développement personnel plutôt qu'une compétition dans la quelle on découvre quels sont les organismes congénitalement les plus aptes: c'est un défi à donner le mieux de so-même, à se dépasser, à acquérir de nouvelles habitudes et de nouveaux comportements pour ne pas être écrasé par ceux qui apprennent mieux et plus rapidement. Une grande partie de ce qu'on a coutume d'appeler darwinisme social (de Spencer à Jack London, de William Graham Sumner à Theodore Roosevelt) n'est qu'une élaboration de cette idée, laquelle, à son tour, n'est qu'une version naturaliste de l'éthique de l'effort et du sacrifice qui a si profondément marqué la pensée occidentale ${ }^{4}$. Une image pré-darwinienne de la nature et de l'homme conditionnait l'acceptation et l'usage, voire la compréhension même de l'idée darwinienne de lutte et pouvait servir à exorciser certaines de ces conséquences morales et sociales. En fait, dans l'interprétation néo-lamarckienne, la lutte pour l'existence, la vraie et la plus importante, était celle qui se livrait contre le milieu. Mais grâce à un renversement curieux - qui en réalité n'en est pas un, sinon de façon apparente-, cette idèe de lutte était souvent utilisée pour prêcher la solidarité et la coopération. L'"effort pour la vie" (une expression du sociologue français René Worms) $)^{5}$ était plus important que la "lutte pour la vie"; chez beaucoup d'auteurs, il signifiait effort de coopération pour améliorer les conditions de vie. Surtout en France, la préférence donnée au lamarckisme sur le darwinisme non seulement permettait, à ceux qui voulaient appliquer la biologie à la société, de réduire l'importance de la lutte, mais encourageait même la cause réformiste. Les partisans des réformes sociales se réclamaient de l'idée lamarckienne de l'influence directe des conditions du milieu sur l'organisme pour affirmer que, en modifiant le milieu social, c'est-à-dire en

3 Ibid., pp. VI-VII.

4 Qu'il me soit permis de renvoyer à mes ouvrages indiqués dans la bibliographie.

5 Worms, R. (1910), p. 114. Worms était un solidariste convaincu: la notion de solidarité, disait-il, était à la racine de tout problème moral, elle était pour l'éthique ce que la notion de valeur est pour l'économie (Philosophie des sciences sociales, Paris, t. III, 1907, pp. 152-153); en France le solidarisme était lié à l'organicisme en sociologie (La sociologie, Paris, 1921, p. 50). 
diffusant l'instruction et en améliorant les conditions de travail ou la qualité de la vie, on pouvait, grâce à l'hérédité des caractères acquis, améliorer les hommes. Lamarckiens étaient aussi la plupart des anthropologues français, ainsi que beaucoup de membres de la "Société d'eugénisme" (fondée en 1912)6 à laquelle appartenait, entre autres, un néo-lamarckien très important que nous allons retrouver plus loin, Edmond Perrier. Les eugénistes français se proposaient d'améliorer la nation en améliorant le milieu plutôt qu'en introduisant des mesures restrictives dans les mariages.

Comme l'a affirmé Yvette Conry, le néo-lamarckisme français est marqué par des instances s'inspirant d'un optimisme bio-socio-politique semblable à celui des Lumières $^{7}$. Un seul exemple suffira. En 1881 fut créé à Paris un "Cercle de gymnastique rationnelle", avec l'appui des lamarckiens Paul Bert et E.-J. Marey. Dans le traitement des déformations, congénitales ou acquises, la gymnastique médicale était conçue comme una application du pouvoir morpho-génétique de l'usage ainsi que des conditions d'existence. "Le corollaire pratique de ce principe", avait écrit l'anthropologue Joseph-Pierre Durand de Gros en 1871, "c'est l'amélioration possible des êtres vivants, et notamment de l'homme, grâce à l'amélioration des milieux"8. Selon un autre lamarckien important, Alfred Giard, il fallait ajouter à l'action primaire de l'éducation le principe selon lequel les impressions subies par l'individu se répercutent sur le produit des glandes génitales. Cela expliquait "le fait que toute une génération accepte avec la plus grande facilité des idées que la génération précédente avait combattues et repoussées"9.

L'un des premiers en France à établir un lien étroit entre lamarckisme et solidarité fut Jean-Louis de Lanessan, le biologiste, publiciste et homme politique qui fut gouverneur en Indochine et ministre de la Marine. Déjà en 1880 il opposa au principe de la lutte pour la vie celui de l'"association pour la lutte", donnant par là l'appui de la biologie à ce "crédo solidariste" dont on a récemment indiqueé qu'il était l'un des thèmes idéologiques dominants de la Troisième République ${ }^{10}$. En biologie et en sociologie, il inspirait une aversion pour la doctrine darwinienne de la lutte pour l'existence, considérée comme la manifestation d'une mentalité individualiste et agressive typiquement britannique, et donc étrangère aux idéaux de la Révolution, à l'esprit français et à la mission civilisatrice de la France. Selon Lanessan ", la tendance à l'association se manifestait même, déjà, chez les minéraux...

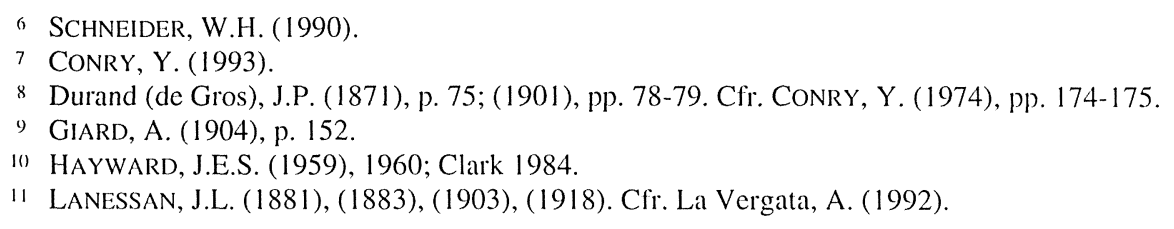


Le crédo solidariste trouvait son expression la plus célèbre dans Solidarité, un petit bouquin publié en 1896 par rien de moins que l'ancien Président du Conseil (il venait de démissionner au mois d'avril), Léon Bourgeois. Bien que le terme ne fût entré que depuis peu d'années dans le vocabulaire de l'actualité, "aujourd'hui", écrivait-il, "le mot de solidarité paraît, à chaque instant, dans le discours et dans les écrits politiques". Bourgeois en appelait à une "doctrine scientifique de la solidarité naturelle". En l'exposant, il donnait une description plutôt lamarckienne que darwinienne de la lutte pour la vie:

\begin{abstract}
"C'est par l'exercice incessant des fonctions développant les organes, par l'adaptation courageuse des organes aux conditions des milieux, que l'individu se développe et se perfectionne; c'est par la suppression des plus faibles et par la survivance et la reproduction des plus forts, que se fixent les qualités utiles de l'espèce et que les êtres qui la composent évoluent vers une forme toujours supérieure"12.
\end{abstract}

La solidarité était la loi fondamentale de la vie:

"Les lois de l'espèce - lois d'hérédité, d'adaptation, de sélection, lois d'intégration et de désintégration- ne sont que les aspects divers de la même loi générale de dépendance réciproque, c'est-à-dire de solidarité, des éléments de la vie universelle"13.

Bien entendu, selon Bourgeois il n'y avait aucune contradiction entre la loi de solidarité et la loi du "libre développement de l'individu": "c'est le concours des actions individuelles dans l'action solidaire qui donne la loi synthétique de l'évolution biologique universelle"14.

Parmi ses sources scientifiques, Bourgeois citait Edmond Perrier, professeur de zoologie au Muséum d'Histoire Naturelle, dont il allait devenir le directeur en 1900. Même dans un ouvrage peu suspect de mélanger science et politique comme sa Faune des côtes de Normandie (1894), celui-ci avait offert les services de la biologie au politiciens, et c'était une biologie lamarckienne basée sur la "loi d'association":

"En établissant que dans le monde vivant, si la lutte est la condition du progrès, comme l'ont si vite appris ceux qui rêvent de bouleversement social, le progrès n'a jamais été réalisé que par l'association des forces individuelles et leur harmonieuse coordination, les sciences naturelles constituent non seulement la plus haute philosophie, mais la seule capable de

12 BOURGEOIS, L. (1896), p. 40.

1.3 Ibid., p. 45 .

14 Ibid., p. 58 


\section{LAMARCKISME ET SOLIDARITÉ}

fournir aux gouvernements les lumières nécessaires pour sonder et guérir les plaies profondes du temps présent"15.

Nous ne nous attarderons pas sur la philosophie biologique de Perrier: on ne peut rien ajouter à ce qu'a écrit la-dessus Claude Blanckaert. Nous nous bornerons à souligner que pour Perrier la "loi d'association" était la loi générale du monde vivant et qu'il la présentait dans un cadre lamarckien. Les principes de Darwin n'étaient qu'un complément de ceux de Lamarck, qui formaient la base inébranlable du transformis$\mathrm{me}^{16}$. Dans la préface à la deuxième édition (1898) de son chef d'oeuvre, Les colonie animales, Perrier écrivait:

\footnotetext{
"...Ce livre établira qu'elles [les sciences naturelles] ne nous prêchent pas seulement la lutte pour la vie; elles nous montrent le succès dans cette lutte, le progrès dans la puissance, résultant de l'association; elles nous enseignent que dans toute association prospère, les éléments associés, tout en gardant les uns vis-à-vis des autres une liberté qui est la condition nécessaire du progrès ${ }^{17}$, demeurent unis par d'incessantes condescendances, et confirment la place toujours plus élevée que prend, parmi les vertus sociales, la pratique de la solidarité"18.
}

L'association est le phénomène le plus général de la nature; la "loi d'association", la loi la plus générale et synthétique: elle unit des individus d'espèce différente, des individus de même espèce, les parties du même organisme. Elle englobe aussi les lois darwiniennes: "Que sont d'ailleurs la lutte pour l'existence et la sélection naturelle, ces principes fondamentaux de la théorie de Darwin, sinon les conséquences rigou-

15 Cité par Bourgeois, L. (1896), pp. 59-60. Selon Marcellin Berthelot, ministre des affaires étrangères du cabinet Bourgeois, la morale devait être basée sur le déterminisme naturel et scientifique. Malheureusement, la notion capitale de solidarité avait été longtemps paralisée par celle de charité chrétienne (Science et morale, Paris, 1897, p. 28).

16 Selon Perrier, la doctrine de Lamarck "n'est pas la rivale, mais la base indispensable de celle de Darwin". "L'origine des formes vivantes, Darwin ne s'en préoccupe pas; que le monde vivant ait commencé par un grumeau de gelée, ou que les principaux types du règne végétal et du règne animal aient paru simultanément, peu lui importe; il s'accommoderait aussi bien des quatre embranchements de $\mathrm{Cu}$ vier que de l'unité du plan de composition de Geoffroy [...] La doctrine nouvelle [celle de Darwin] s'attaque à d'autres problèmes que celle de Lamarck; elle pourrait lui faire suite si celle-ci avait atteint le but qu'elle se proposait, mais elle ne saurait la remplacer. Il n'y a donc pas, comme on le fait quelquefois, à opposer le darwinisme au lamarckisme; ce sont tout au plus deux doctrines qui se juxtaposent sans qu'il existe nécessairement entre elles une opposition ou même une superposition" (PERRIER, E. (1893), pp. 487, 491, 492). Et ainsi de suite. Sur Perrier voir Blanckaert 1979.

17 Et qui fait que les composants élémentaires des organismes, les plastides et les mérides, sont soumis eux aussi à la concurrence vitale et à la sélection naturelle.

18 PERrier, E. (1898), p. XXXII. 
reuses de l'association forcée dans laquelle sont maintenus sur le globe tous les êtres vivants?"19 Tout organisme est une collection de protozoaires. La solidarité en politique a donc sa base naturelle et sa justification dans le "polyzoïsme" répandu dans le monde vivant ${ }^{20}$.

Le mot "polyzoïsme" n'avait pas été inventé par Perrier, mais (avec le terme correspondant de "polypsichisme") par l'anthropologue et physiologiste Joseph-Pierre Durand (de Gros) ${ }^{21}$. Mais ce qui est plus intéressant pour notre discussion, c'est que Durand, qui aimait les néologismes, avait aussi inventé un mot bizarre dont il se servait pour désigner la doctrine bio-sociologique qu'il détestait: struggleforlifisme ${ }^{22}$. Le struggle for life, écrivait Durand, est, même chez les animaux, le caractère des agglomérations primitives, confuses, non-orga-nisées. Le genre humain a progressivement abandonné la phase primordiale de la lutte et marche vers son contraire, le "collectivisme organique". Et déjà la lutte entre les groupes organisés est une forme supérieure de lutte. Mais "nos purs darwinistes sociologiques" s'obstinent à ne voire, dans le futur comme dans le passé, dans la nature comme dans la société, que "luttes fratricides, dévastations et massacres"23. L'opposition à l'interprétation darwinienne, et surtout néo-darwinienne, de la lutte pour l'existence, allait de pair, chez Durand comme chez tant d'autres, avec celle à la toute-puissance de la sélection et avec la défence à outrance de l'hérédité des caractères acquis par des raisons morales et so-

19 Ibid., p. 714. Il faut reconnaître que cette formule est très peu satisfaisante. Quoi qu'il en soit, pour Perrier la loi d'association n'est pas seulement une loi d'aggrégation, mais aussi une loi morphogénétique, elle est elle-même une cause de variation héréditaire: "Par cela seul qu'elle réalise des conditions d'existence particulières et d'une variété exceptionnelle autour des individus qui s'y soumettent, la vie sociale, quelle que soit sa forme, détermine chez ces individus des variations nombreuses et devient par conséquent une cause puissante de diversifications dans le Règne animal. Qu'elle unisse des individus d'espèce différente, elle produit les races et les variétés innombrables qui caractérisent les espèces parasites, commensales ou neutralistes; qu'elle unisse des individus de même espèce, elle produit chez eux tous les phénomènes qui ont été groupés sous la dénomination générale de polymorfisme; que ces individus conservent entre eux des liens physiologiques, comme dans les colonies, leur société se transforme en organisme grâce à la puissance qui résulte pour elle de la division du travail physiologique entre ses membres; ella commence ainsi une nouvelle évolution. Que sont d'ailleurs etc..." (ibid., pp. 713-714).

20) Aussi n'est-il pas étonnant de lire que "les syndicats ne sont nullement d'invention humaine; il tempèrent, dans le règne animal, les effets de la lutte pour la vie"... (PERRIER, E. (1929), p. 238). (Mais Perrier se sert du terme avec une certaine liberté: il dit, par exemple, que "les individus de l'un des sexes forment avec ceux de l'autre sexe un syndicat nécessaire à la continuité de la vie sur le Globe", ibidem).

21 DuRAnd (de Gros), J.-P. (1867). Perrier reconnut à Durand la paternité officielle du concept et du terme de polyzoïsme lors de la séance de l'Académie des Sciences du 4 mars 1895. Par contre, Durand accusa Claude Bernard d'avoir plagié, dans son discours de réception a l'Académie Française, la théorie du polyzoïsme.

22 Durand (de Gros), J.-L. (1901). Le terme fut repris par Alphonse Daudet dans sa pièce La lutte pour la vie (1889; publiée en 1890).

2.3 DuRAnd (de Gros), J.-L. (1901), pp. 84, 86. 
ciales. Durand attaquait Weismann ${ }^{24}$ et se servait au cours de cette attaque des arguments traditionnels qu'avaient popularisés les lamarckiens tels que Perrier: la sélection à elle seule ne suffit pas; elle ne crée pas, elle agit sur des espèces qui existent déjà, mais n'explique pas d'où celles-ci viennent. "Si on retire l'explication de Lamarck, qui les tire d'une modification d'espèces antérieures sous l'action de causes naturelles, il faut revenir à la Bible". Bref, le véritable transformisme, c'est le lamarckisme; le darwinisme (c'est-à-dire la lutte et de la sélection) est un mécanisme secondaire de finition. Mais c'est surtout la négation de l'hérédité de l'acquis qui est à la base des conséquences les plus horribles du struggleforlifisme.

\footnotetext{
"La thèse folle de l'intrasmissibilité des caractères acquis paraît être un expédient inventé pout donner une base biologique au dogme aristocratique de la nouvelle anthroposociologie, qui, avec une franchise dont il faut lui tenir compte, pose carrément en principe le rétablissement des castes au profit d'une prétendue race aryenne, mais qui est avant tout imaginaire."

Par contre,

"le principe transformiste ou évolutionniste proclamé par Lamarck est essentiellement égalitaire, et il autorise et encourage toutes les espérances d'amélioration, de perfectionnement et de relèvement." 25
}

Racisme et culte de la force brutale étaient donc le résultat de l'adoption unilatérale du principe de la lutte. On peut bien imaginer l'usage qu'on fit de ces idées pendant la première guerre mondiale. La doctrine de la force imaginée par Darwin et renforcée par ses disciples, soutenait Lanessan, était la doctrine dont s'était nourri le germanisme agressif qui avait déchaîné la catastrophe ${ }^{26}$. La guerre, pour Lanessan comme pour tant d'autres, c'était la faute aux allemands et au "darwinisme à l'allemande"27. Curieusement, on étendait un voil sur les darwinistes sociaux anglais et américains; mais on ne peut pas parler mal de ses alliés... Quoi qu'il en soit, dans le grand affrontement entre France et Allemagne, présenté comme un affrontement entre civilisation et barbarie, les biologistes ne furent en reste par rapport à personne,

\footnotetext{
${ }^{24}$ Qu'il appelle "Weisemann" et qu'il montre d'ailleurs ne pas avoir bien compris: ibid., pp. 92.94, 96. Curieusement, Durand n'est pas le seul à se tromper sur le nom du savant allemand: cfr. PERRIER, E. (1893), qui écrit "Weissmann" (pp. 507.508 etc.).

25 Ibid., p. 97, 99. Les discussions sur le caractère aristocratique ou démocratique du darwinisme n'étaient pas une spécialité française: qu'il suffise de rappeler ici le débat de 1876 entre Virchow et Haeckel, dans lequel intervint Thomas Henry Huxley.

26 LANESSAN, J.-L. (1918). Cfr. La Vergata 1992

27 Colajanni, N. (1884), (1906). Cfr. La Vergata 1994. 


\section{ANTONELLO LA VERGATA}

et la doctrine néo-lamarc-kienne de la solidarité devint une puissante arme défensive et offensive. Aux armes, lamarckiens! Le Muséum, lui aussi, s'en va-t-en guerre.

Il y était déjà allé lors du conflit franco-allemand, quand il avait subi un borbardement (95 obus entre le 8 et le 25 janvier 1870 - voire figure - : aucune victime, des pertes légères dans les parties végétale et conchyliologique, une perruche tuée) et Jean-Louis-Armand de Quatrefages, professeur d'anthropologie au Muséum de 1855 à 1892, avait soutenu que la race prussienne était anthropologiquement étrangère à l'Allemagne et, à proprement parler, n'existait pas: c'était une race bâtarde de Finnois et de Slaves, brachycéphale, atavistique et sauvage ${ }^{28}$. A la même année remonte aussi la première accusation de bellicisme biologique portée aux Allemands. Ernest Renan s'était élevé contre ces "naturalistes allemands qui ont la prétention d'appliquer leur science à la politique" et "soutiennent, avec une froideur qui voudrait avoir l'air d'être profonde, que la loi de la destruction des races et de la lutte pour la vie se retrouve dans l'histoire, que la rasse la plus forte chasse nécessairement la plus faible, et que la race germanique, étant plus forte que la rece latine et la race slave, est appelée à les vaincre et à se les subordonner"29.

Renan avait renchéri dans sa lettre à David Friedrich Strauss du 15 septembre 1871:

\footnotetext{
"Notre politique, c'est la politique du droit des nations; la vôtre, c'est la politique des races: nous croyons que la nôtre vaut mieux. La division trop accusée de l'humanité en races, outre qu'elle repose sur une erreur scientifique, très peu de pays possédant una race vraiment pure, ne peut mener qu'à des guerres d'extermination, à des guerres "zoologiques", permettez-moi de le dire, analogues à celles que les diverses espèces de rongeurs ou de carnassiers se livrent pour la vie. Ce serait la fin de ce mélange fécond, composé d'éléments nombreux et tous nécessaires, qui s'appelle l'humanité. Vous avez levé le drapeau de la politique ethnographique et archéologique en place de la politique libérale; cette politique vous sera fatale ${ }^{30}$.
}

28 QuATREFAGES, J.-L.-A. (1871). En plus, la race prussienne aurait été d'origine non aryenne, mais turanienne, comme les Lapons, les Bretons et les Basques. A Quatrefages répondirent Virchow et Bastian. Cfr. Pick 1993, pp. 93-95.

29 RENAN, E. (1947), p. 434

30 Ibid., p. 457. Mais il ne faut pas oublier que, quand le sort du conflit n'était pas encore décidé. Renan s'était adressé à ses compatriotes dans des termes bien différents: "Si la sottise, la négligence, la paresse, l'imprévoyance des Etats n'avaient pour conséquence de les faire battre, il est difficile de dire à quel degré d'abaissement pourrait descendre l'espèce humaine. La guerre est de la sorte une des conditions du progrès, le coup de fouet qui empêche un pays de s'endormir, en forçant la médiocrité satisfaite d'elle-même à sortir de son apathie. L'homme n'est soutenu que par l'effort et la lutte. La lutte contre la nature ne suffit pas; l'homme finirait, au moyen de l'industrie, par la réduire à peu de chose. La lutte des races se dresse alors. Quand une population a fait produire à son fonds tout ce qu'il peut produire, elle s'amollirait, si la terreur de son voisin ne le réveillait; car le but de l'humanité n'est pas de jouir; acquérir et créer est oeuvre de force et de jeunesse: jouir est de la décrépitude. La crainte de la conquete est ainsi, 
Pendant la guerre mondiale, les professeurs du Muséum sont de nouveau en première ligne. Ils contribuent à une vaste littérature adressée contre la science allemande, ou contre ce qu'un autre lamarckien important, Félix Le Dantec, appelle "le bluff de la science allemande"31. Celle-ci est chaque fois qualifiée de "touffue", "lourde", "fuligineuse", "prolixe", "obscure", "froide", "inexo-rable", "brutale", "immorale". Voilà donc Stanislas Meunier, professeur de géologie au Muséum, critiquer la géologie à la prussienne, "froide et compasseée, prompte à se manifester avec une allure anguleuse que traduit, avec une brutalité légendaire, la discipline militaire d'outre-Rhin".

"Elle rêve de distribuer les choses de la nature dans les cadres rigides d'une classification sans appel, elle prétend établir la série des formations géologiques, inflexiblement immatriculées sous des numéros définitifs, comme se passe la revue d'un régiment de la Garde, défilant au pas de l'oie." 32

Volià Marcellin Boule, professeur de paléontologie au Muséum, réitérer le jugement de son maître Quatrefages en y ajoutant des arguments tirés de l'evolution que celui-là n'avait pas pris en considération: la race allemande se trove dans la condition des espèces destinées à une disparition rapide à cause de leur gigantisme ou de leur spécialisation exagérée dans une seule direction, c'est-à-dire à cause d'une perte de souplesse et de capacité d'adaptation.

"L'évolution allemande s'est faite, elle aussi, plutôt dans le sens qui tue que dans celui qui vivifie. La "Kultur", qui en est résultée, doit périr, victime d'une spécialisation exclusive, du gigantisme de sa force matérielle, de l'atrophie de son sens moral."33

Par contre, le peuple français...

"Toujours épris de mesure et d'harmonie, il a su, heureusement, conserver, avec l'équilibre de ses facultés, cette plasticité organique, cet esprit d'initiative individuelle [voilà des mots clés du lamarckisme bio-social!] qui sont comme les marques mêmes de sa vigoureuse constitution et qui lui ont permis de s'adapter facilement, avec succès, aux conditions nouvelles d'existence et de lutte que lui imposait l'agression des barbares." $3 .-4$

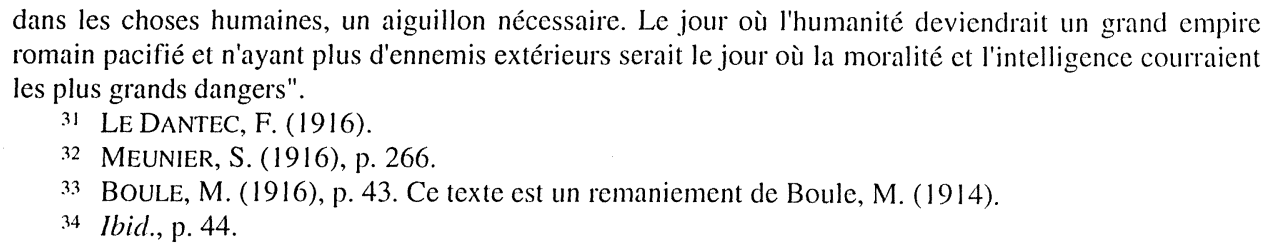


Et tout cela s'explique, bien entendu, "par les lois naturelles de notre grand Lamarck sur l'influence du milieu et sur l'hérédité".

"Dès les temps paléolithiques, notre pays, aux clairs paysages, constitua un foyer de progrès au regard des contrées voisines et notamment des marécages brumeux de l'Europe centrale. Les reliques de ce lointain passé, dont le Allemands se montraient envieux au point de nous les faire voler par leus émissaires, prouvent que ces ancêtres avaient déjà le culte de la beauté, le souci de leur perfectionnement et même un certain sentiment de l'idéal. Ils furent les premiers pionniers de la vraie culture. Leur intelligence et leur bravoure vinrent à bout des bêtes redoutables qui leur disputaient l'abri des cavernes. Façonnés par les mêmes milieux, nourris par le même sol, baignés dans la subtilité de la même atmosphère, vivifiés par le même soleil, nos modernes "poilus" ont de qui tenir. Unis à leurs alliés, tous défenseurs des justes causes, tous soldats de la liberté et du droit, ils ont retrouvé leurs énergies ancestrales, et ils viendront certainement à bout du monstre germanique dont l'évolution a mal tourné. Et ce sera, une fois de plus, le triomphe de l'esprit sur la matière." 35

Perrier, quant à lui, intervint par un grand nombre d'articles et par un livre, France et Allemagne (1915), où il faisait son devoir de patriote en critiquant les aspects dangereux de la science et de la civilisation allemande (notamment Haeckel) aussi bien qu'en affirmant ses idées sur la solidarité et l'adaptation par l'effort. Il ne le déclarait pas haut, mais l'infra-texte du passage qui suit est clair: le néo-darwinisme est une doctrine de la toute-puissance de la lutte et donc de la guerre; le lamarckisme est une doctrine de la solidarité et de l'effort sur soi-même, donc une philosophie de la paix.

\footnotetext{
"Si d'une part la lutte pour la vie a joué un certain rôle dans le progrès matériel des êtres vivants, d'autre part elle a contribué à ce processus seulement dans les détails des formes organiques. Une plus large coordination de tous les grands faits de la biologie nous permet, en revanche, d'établir que les grands types du règne animal se sont constitués dans la paix, grâce aux efforts constants des animaux sur eux-mêmes, grâce à la tension constante de leurs facultés pour triompher des conditions défavorables dans lesquelles certains étaient condamnés à vivre"36.
}

Perrier écrivit aussi la préface à un livre dont le titre est tout un programme, $L a$ science des civilisés contre la science des Allemands (1916). Entre une invective et l'autre, l'auteur, lebiochimiste Pierre-Jean Achalme, directeur du laboratoire colonial du Muséum, nous offre d'ultérieures démonstrations de la façon dont on pouvait combiner la critique de l'esprit allemand et la revendication du lamarckisme. Selon

35 Ibid., pp. 44-45.

36 PERrier, E. (1915), p. 103. 
Achalme, les théories de Weismann fournissent un example de ce que l'illusion des mots hypnotise les Allemands et que l'invention d'un terme nouveau et difficile constitue pour eux une explication suffisante des phénomènes. Pour les savants d'outre-Rhin il s'agissait de "saboter le monument scientifique de l'évolution naturelle qui, fondée par Aristote, Descartes, Buffon, construit par Lamarck, Etienne Geoffroy Saint-Hilaire, Darwin, ne contient pas une pierre tudesque, bien qu'E. Haeckel ait prétendu innover en exploitant en Allemagne les brevets Darwin"37. Par toute réponse, Weismann a inventé les "plasmas ancestraux", les "ides", les "idantes", les "biophores"...

"La réalité doit s'humilier devant les mots, sortis de l'imagination de Weismann et qui deviennent des dogmes intangibles. Le délire de l'abstraction peut-il être poussé plus loin? Et c'est avec de pareilles armes que l'on bombarde la forteresse de Lamarck, dont la solidité est à l'abri de semblables attaques." 38

C'est que les Allemands ont très peu compris aux principes directeurs de l'évolution organique, et cela a eu des

conséquences qui vont au-delà du domaine biologique.

\begin{abstract}
"Cette incompréhension a exercé sur l'esprit germanique une influence qui n'est peut-être pas étrangère aux événements actuels. Sans voir autre chose que les mots, la Prusse ou plutôt l'Allemagne prussianisée, s'est hypnotisée sur le deuxième principe de Darwin: la lutte pour la vie. Dans la naïveté féroce de ses conceptions, elle l'a traduit immédiatement par l'oppression et la destruction du plus faible par le plus fort, et a cherché ainsi dans la philosophie naturelle un argument ou plutôt une excuse pour les tendances instinctives vers lesquelles la poussait son hérédité sauvage. Son culte de la Force, les élucubrations des Treitschke, des Waldersee, des Bernhardi, des Nietzsche et autres apologistes du crime, ne sont que des commentaires indirects du deuxième principe de Darwin." ${ }^{39}$
\end{abstract}

Le conflit entre la France et l'Allemagne est donc, entre autres, un conflit entre Lamarck et Darwin, ou du moins un Darwin interprété unilatéralement. Les doctrines du premier ne sont pas seulement des principes inspirateurs de la lutte contre la barbarie, mais aussi une arme dans cette lutte; et ce sont les principes lamarckiens euxmêmes qui vont décider qui va gagner: réciproquement, la défaite de l'Allemagne sera la preuve définitive de la vérité des théories de Lamarck.

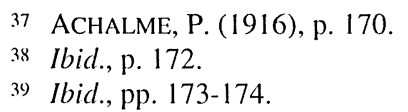




\begin{abstract}
"Mais la Violence n'est pas la Force; aussi l'Allemagne paiera sa méconnaissance des principes de Lamarck. L'ambiance joue le rôle principal et celui qui vaincra ne sera pas celui qui aura à sa disposition les moyens les plus violents, mais celui dont les armes seront les mieux adaptées au champ de bataille. Suivant qu'elle se produira sur la terre ou dans l'eau, nous pourrons prévoir à coup sûr l'issu de la lutte entre un tigre et un crocodile. On n'est jamais assez fort pour triompher du milieu, quand même. C'est lui qui triomphe et transforme en fossiles les espèces inadaptées. A la défiance et à la haine qui l'entoiurent, l'Allemagne devra bien reconnaitre que les forces matérielles ne constituent pas à elles seules la Force, et que, dans un milieu civilisé, les forces morales [...] sont, pour les nations, les armes les meilleures dans la lutte pour la vie."40
\end{abstract}

Quelle explication (je n'ose pas dire justification) qu'on présente d'affirmations comme celles-ci, nous ne pouvons pas passer sous silence des faits que nos lamarckiens patriotes semblaient avoir oublié:

1) Il y avait eu des partisans importants de l'entraide aussi en Allemagne, à partir de Ludwig Büchner. Le darwinisme popularisé en Allemagne par des écrits incroyablement répandus tels que ceux de Wilhelm Bölsche était un darwinisme où la coopération n'avait certainement pas une place moindre que la lutte. Selon la version du darwinisme social très répandue dans la classe ouvrière grâce à l'activité d'un Bebel ou d'un Kautsky, tant la concurrence effrénée que la guerre appartenaient à une phase primitive de l'évolution sociale. Enfin, dans une vaste littérature, la métaphore de l'organisme social était employée pour souligner la nécessité non de la concurrence, mais de la coopération entre les membres de la société, ou de leur subordination aux intérêts généraux. L'influence des doctrines de l'"état cellulaire" de Virchow ou d'Oscar Hertwig allait dans la même direction. Enfin, nous savons aujourd'hui qu'il y eut des lamarckiens pacifistes aussi dans les pays de langue allemandes ${ }^{41}$.

2) Une très large partie d'intellectuels allemands soutint la nécessité de la guerre de 1914 non au nom du darwinisme, qu'ils méprisaient en tant que doctrine matérialiste, utilitariste est typiquement britannique (donc étrangère aux valeurs de la véritable tradition germanique), mais de valeurs idéaux (Max Scheler) ou d'un organicisme anti-matérialiste (Oswald Spengler) ${ }^{42}$.

40) Ibid., p. 174. Et la force matérielle que l'Allemagne met sur le terrain n'est elle-même qu'une application militaire d'inventions faites, pour des fins pacifiques, par des Français et des Anglais, et jamais par des Allemands! (pp. 174-178).

41 Voire par exemple les écrits recueillis dans Kammerer 1918. Kammerer, lamarckien et défenseur acharné de l'hérédité de l'acquis, avait opposé les principes de l'aide réciproque et de la symbiose à celui de la lutte en plusieurs travaux publiés avant la guerre. Il n'était pas allemand, mais autrichien, bien sûr, mais il appartenait quand même à cette "Austro-Bochie" contre les savants de laquelle tonnait un autre lamarckien, Delage, Y. (1916). Sur le darwinisme social en Allemagne (et en Autriche), voire Kelly, A. (1981); Rupp-Eisenreich, B. (1992).

42 Cfr. La Vergata, A. (1994). 
3) Les dangers de l'interprétation "allemande" du darwinisme ne consistait pas dans l'éloge de la lutte individuelle, mais, au contraire, dans l'attribution d'une importance dominante à la lutte entre les groupes (races ou nations) et à la subordination des intérêts de l'individu à ceux de l'ensemble (de la race ou de la nation). Ce n'était pas toujours du darwinisme, et même pas toujours du biologisme, et même dans les cas où c'était du biologisme, il en était un largement revêtu d'idéalisme. Le culte du Volk peut même être vu comme une version spéciale de l'idéologie du solidarisme. Paix et solidarité à l'intérieur de la nation (et abolition de la lutte de classe), concurrence et, si nécessaire, guerre à l'extérieur: c'était le programme adopté avec enthousiasme tant en Allemagne qu'en Angleterre par les théoriciens de ce qu'on a défini "impérialisme social"43.

4) Les lamarckiens patriotes passaient sous silence toute une foule d'auteurs anglais et américains qui avaient soutenu une version du darwinisme social non moins agressive quelle celle qu'on attribuait aux allemands en tant que tels.

Mais en temps de guerre les oublis et les refoulements sont normaux...

Et en temps de paix? Les apologistes de la guerre ont toujours insisté sur ce qu'en guerre les hommes (y compris les savants) révèlent leurs meilleures qualités: courage, abnégation, loyauté, etc. Mais les pacifistes ont toujours eu beau jeu de répliquer qu'en guerre les hommes révèlent leurs pires qualités (et cela aussi est vrai pour les savants). En temps de guerre tout semble malheureusement être admis ${ }^{44}$. Faire la caricature des idées de ses adversaires est un procédé difficilement justifiable, bien que normal, en temps de guerre, mais il est absolument inacceptable en temps de paix. Cependant, les outils et les techniques idéologiques dont on se sert en guerre sont fabriqués pendant la paix. De ce danger nul n'est exempt, qu'il soit scientifique ou commun mortel, qu'il soit au chômage intellectuel ou qu'il ait la chance de travailler dans une institution scientifique prestigieuse.

4.3 Semmel, B. (1960).

${ }_{44}$ Et -mais est-il nécessaire de le dire?- aucune nations n'est exempte de ce danger. 
ANTONELLO LA VERGATA

\section{BIBLIOGRAPHIE}

ACHALme, P. (1916), La science des civilisés et la science allemande. Avec une préface de M. Edmond Perrier, président de l'Académie des Sciences, et une réponse du professeur Ostwald, Paris, Payot.

Blanckaert, C. (1979), «Edmond Perrier et l'étiologie du "polyzoïsme organique"», Revue de Synthèse, 100, pp. 353-376.

BOULE, M. (1914), «La guerre», L'Anthropologie, 25, pp. 575-580.

Boule, M. (1916), La guerre et la paléontologie, in Petit et Leudet, pp. 33-45.

Bourgeols, L. (1896), Solidarité, Paris, A. Colin.

Clark, L.L. (1984), Social Darwinism in France, The University of Alabama Press.

Colajanni, N. (1884), Il socialismo, Catania, F. Tropea.

Colajanni, N. (1906), Latini e Anglo-sassoni (Razze inferiori e razze superiori), Roma, presso la Rivista Popolare.

CONRY, Y. (1974), L'introduction du darwinisme en France au XIXe siècle, Paris, Vrin.

ConRY, Y. (1993), «Commment a-t-on pu être néo-lamarckien en France (1843-1930)?, Nuncius, VIII, (1).

Delage, Y. (1916), «Histoire naturelle du Doctus Bochensis», dans Petit et Leudet, pp. 99-115.

Durand (de Gros), J.-L. (1867), «Polyzoösme ou pluralité animale dans l'homme», Bulletin de la Société d'anthropologie de Paris, 2e série, pp. 600-616.

DURAND (de Gros), J.-P. (1871), Les origines animales de l'homme éclairées par la physiologie et l'anatomie comparatives, Paris, Baillière, 1871.

Durand (de Gros), J.-P. (1901), Questions de philosophie morale et sociale, Paris, Alcan.

GIARD, A. (1904), Controverses transformistes, Paris, Naud.

HAYWARD, J.E.S. (1959), «Solidarity: the social history of an idea in nineteenth century France», International Review of Social History, IV, pp. 261-284.

HAYwARD, J.E.S. (1961), «The official philosophy of the french Third Republic: Léon Bourgeois and solidarism», International Review of Social History, VI, pp. 19-48.

KAMmerer, P. (1918), Einzeltod, Völkertod, biologische Unsterblichkeit und andere Mahnworte aus schwerer Zeit, Wien, Anzengruber-Verlag Brüder Suschitzky.

Kelly, A. (1981), The descent of Darwin: the popularization of Darwinism in Germany, 1860 1914, Chapel Hill, University of North Carolina Press.

LANESSAN, J.-L. de (1881), Etudes sur la doctrine de Darwin: la lutte pour l'existence et l'"association pour la lutte", Paris, O.Doin (original dans la Revue internationale des sciences biologiques, VII, pp. 289-329). 


\section{LAMARCKISME ET SOLIDARITÉ}

LANESSAN, J.-L. de (1883), Le transformisme, évolution de la matière et des êtres vivants, Paris, O. Doin.

LANESSAN, J.-L. de (1903), La lutte pour l'existence et l'évolution des sociétés, Paris, Alcan.

LANESSAN, J.-L. de (1918), L'idéal moral du matérialisme et la guerre, Paris, F. AlCAN.

La Vergata, A. (1982), «Biologia, scienze umane e "darwinismo sociale": considerazioni contro una categoria storiografica dannosa», Intersezioni, II, pp. 77-97.

La Vergata, A. (1990a), L'equilibrio e la guerra della natura. Dalla teologia naturale al darwinismo, Napoli, Morano.

LA Vergata, A. (1990b), «Il lamarckismo fra riduzionismo biologico e migliorismo sociale», Intersezioni, X, pp. 495-516.

La Vergata, A. (1992), «Les bases biologiques de la solidarité», dans Tort, P. (ed.), Darwinisme et société, Paris, Presses Universitaires de France, pp. 55-87.

La Vergata, A. (1995), «La lutte et l'effort», dans Blanckaert, C.; Fischer, J.-L. et Rey, R. (eds.) Essais en mémoire de Jacques Roger, Paris, Klincksieck, pp.241-252.

La Vergata, A. (1994), «Evolution and war», Nuncius, IX, (1).

Le Dantec, F. (1916), «Le bluff de la science allemande», dans Petit, G. et Leudet, M., pp. 243-250.

Meunier, S. (1916) La géologie à la prussienne, dans Petit, G. et Leudet, M., pp. 263-273.

PERRIER, E. (1893), «Lamarck et le transformisme actuel», dans Centenaire de la fondation du Muséum d'Histoire Naturelle, Paris, Imprimerie Nationale, pp. 470-522.

Perrier, E. (1898), Les colonies animales, 2e édition, Paris, Masson [première édition 1881].

Perrier, E. (1915), France et Allemagne, Paris, Payot.

Perrier, E. (1929), A travers le monde vivant, Paris, Flammarion.

Petit, G. et Leudet, M. (1916), Les Allemands et la science. Préface de M. Paul Deschanel, Paris, F. ALCAN.

PICK, D. (1993), War machine. The rationalization of slaughter in the modern age, New Haven and London, Yale University Press.

Quatrefages, J.-L.-A. de (1871), La race prussienne, Paris, Hachette.

RENAN, E. (1947), «La réforme intellectuelle et morale [1871]», dans Oeuvres complètes d'Ernest Renan, édition définitive établie par Henriette Psichari, t. I, Paris, Calmann-Lévy.

Rupp-EisenREich, B. (1992), «Le darwinisme social en Allemagne», dans Darwinisme et société, sous la direction de P. Tort, Paris, Presses Universitaires de France, pp. 169-236.

SCHNEIDER, W.H. (1990), «The eugenics movement in France, 1890-1940», dans The wellborn science. Eugenics in Germany, France, Brazil, and Russia, edited by Mark B. Adams, New York and Oxford, Oxford University Press, pp. 69-109. 
ANTONELLO LA VERGATA

Semmel, B. (1960), Imperialism and social reform. English social-imperial thought, 18951914, London, Allen \& Unwin.

Tocquet, R. (1953), L'entraide dans le monde des animaux et des plantes. Introduction de Louis Marin, Paris, Dunod.

Worms, R. (1910), Les principes biologiques de l'évolution sociale, Paris, Giard et Brière. 